\title{
Loss of renal SNX5 results in impaired IDE activity and insulin resistance in mice
}

\author{
Fengmin $\mathrm{Li}^{1}$ • Jian Yang ${ }^{2,3} \cdot$ Van Anthony M. Villar ${ }^{2,4}$ - Laureano D. Asico ${ }^{2,4}$. \\ Xiaobo $\mathrm{Ma}^{4}$ • Ines Armando ${ }^{2,4}$ - Hironobu Sanada ${ }^{5}$ Minoru Yoneda ${ }^{5}$. \\ Robin A. Felder ${ }^{6}$ Pedro A. Jose $\mathrm{S}^{2,4,7}$ - Xiaoyan Wang ${ }^{2,4}$
}

Received: 6 April 2017 / Accepted: 2 October 2017 / Published online: 28 October 2017

(C) Springer-Verlag GmbH Germany 2017

\begin{abstract}
Aims/hypothesis We hypothesised that renal sorting nexin 5 (SNX5) regulates the insulin-degrading enzyme (IDE) and, thus, circulating insulin levels. We therefore studied the dynamic interaction between SNX5 and IDE in human renal proximal tubule cells (hRPTCs), as well as in rat and mouse kidneys.

Methods The regulation of IDE by SNX5 expressed in the kidney was studied in vitro and in vivo. Snx5 or mock siRNA was added to immortalised hRPTCs (passage $<20$ ) in culture or selectively infused, via osmotic mini-pump, into the remnant kidney of uninephrectomised mice and rats.

Results SNX5 co-localised with IDE at the plasma membrane and perinuclear area of hRPTCs and in the brush border membrane of proximal tubules of human, rat, and mouse kidneys. Insulin increased the co-localisation and co-immunoprecipitation of SNX5 and IDE in hRPTCs. Silencing SNX5 in hRPTCs decreased IDE expression and activity. Renal-selective silencing of Snx5 (SNX5 pro-
\end{abstract}

Fengmin Li and Jian Yang contributed equally to the work and should be considered as co-first authors.

Electronic supplementary material The online version of this article (https://doi.org/10.1007/s00125-017-4482-1) contains peer-reviewed but unedited supplementary material, which is available to authorised users.

Xiaoyan Wang

xywang@gwu.edu

1 Department of Physiology and Biophysics, Georgetown University Medical Center, Washington, DC, USA

2 Division of Nephrology, Department of Medicine, University of Maryland School of Medicine, Baltimore, MD, USA

3 Department of Nutrition, The Third Affiliated Hospital of Chongqing Medical University, Chongqing, People's Republic of China tein: $100 \pm 25$ vs $29 \pm 10, p<0.05$ [\% of control]) in $\mathrm{C} 57 \mathrm{Bl} / 6 \mathrm{~J}$ mice decreased IDE protein $(100 \pm 13 \mathrm{vs}$ $57 \pm 6, p<0.05$ [\% of control]) and urinary insulin excretion, impaired the responses to insulin and glucose, and increased blood insulin and glucose levels. Spontaneously hypertensive rats (SHRs) had increased blood insulin and glucose levels and decreased renal SNX5 $(100 \pm 27$ vs $29 \pm 6, p<0.05[\%$ of control]) and IDE (100 \pm 5 vs $75 \pm 4, p<0.05$ [\% of control]) proteins, compared with normotensive WistarKyoto (WKY) rats. Kidney Snx5-depleted WKY rats also had increased blood insulin and glucose levels. The expression of SNX5 and IDE was decreased in RPTCs from SHRs and hypertensive humans compared with cells from normotensive volunteers, indicating a common cause for hyperinsulinaemia and hypertension.

Conclusions/interpretation Renal SNX5 positively regulates IDE expression and function. This study is the first to demonstrate the novel and crucial role of renal SNX5 in insulin and glucose metabolism.
4 Division of Renal Diseases and Hypertension, Department of Medicine, The George Washington University, Walter G. Ross Hall, Suite 740-C, 2300 I Street, N.W., Washington, DC 20037, USA

5 Division of Health Science Research, Fukushima Welfare Federation of Agricultural Cooperatives, Fukushima, Japan

6 Department of Pathology, The University of Virginia, Charlottesville, VA, USA

7 Department of Pharmacology and Physiology, The George Washington University, Washington, DC, USA 
Keywords Human · Insulin-degrading enzyme $\cdot$ Mouse Rat $\cdot$ Renal proximal tubule cell $\cdot$ Sorting nexin 5

$\begin{array}{ll}\text { Abbreviations } \\ \text { FRET } & \text { Fluorescence resonance energy transfer } \\ \text { GAPDH } & \text { Glyceraldehyde 3-phosphate dehydrogenase } \\ \text { hRPTC } & \text { Human RPTC } \\ \text { IDE } & \text { Insulin-degrading enzyme } \\ \text { RPTC } & \text { Renal proximal tubule cell } \\ \text { RT-qPCR } & \text { Reverse transcriptase-quantitative real-time PCR } \\ \text { SHR } & \text { Spontaneously hypertensive rat } \\ \text { shRNA } & \text { Small hairpin RNA } \\ \text { siRNA } & \text { Small interfering RNA } \\ \text { SNX } & \text { Sorting nexin } \\ \text { WKY } & \text { Wistar-Kyoto }\end{array}$

\section{Introduction}

Hyperinsulinaemia is caused by a variety of conditions, one of which is related to increased insulin secretion, as seen in congenital hyperinsulinism, polycystic ovary disease, and metabolic syndrome and after intake of certain drugs [1-4]. Hyperinsulinaemia can also occur because of decreased insulin degradation, as seen in Goto-Kakizaki rats and humans with polymorphisms of the gene encoding insulin-degrading enzyme (IDE) [5-8]. Insulin degradation is also decreased in visceral and subcutaneous fat of diabetic individuals [9]. Rats fed with cafeteria food develop insulin resistance and display increased IDE expression and activity in the liver but not in the adipose tissue and kidney [10].

Insulin is cleared from the body by several organs but the primary sites of insulin clearance are the liver and kidney. About 50\% of portal insulin is removed during the first pass through the liver. The kidney is the major site of the clearance of insulin from the systemic circulation, removing $50 \%$ of circulating insulin [11-13]. Renal proximal tubule cells (RPTCs) are involved in the renal degradation of circulating insulin; the degradation occurs in endosomes (e.g. lysosomes), as reported in the liver [13, 14]. Protein disulfide isomerase and cathepsin D are involved in insulin metabolism but IDE is the major enzyme responsible for degrading insulin $[15,16]$; Ide null mice display hyperinsulinaemia and glucose intolerance [17].

IDE is upregulated by insulin; in hippocampal neurons, insulin increases IDE levels. Treatment of hippocampal neurons with the phosphoinositide 3-kinase inhibitors wortmannin and LY 294002 blunts the upregulation of IDE caused by insulin [18]. By contrast, all trans-retinoic acid decreases IDE expression and activity in several neuroblastoma cell lines [16]. However, the renal mechanisms involved in the regulation of IDE expression are not known.
The sorting nexin (SNX) family consists of a diverse group of cytoplasmic- and membrane-associated proteins that regulate intracellular trafficking $[19,20]$. An increasing number of studies have shown that SNXs are associated with diseases in which endosomal function is adversely perturbed, such as hypertension [21, 22]. The SNX5 gene maps at chromosome 20p11; a susceptibility quantitative trait locus for high fasting plasma insulin and HOMA-IR has been reported to be located at chromosome 20p in non-diabetic Chinese individuals [23]. In overexpression studies, SNX5 was found to interact with and decrease the degradation of the epithelial growth factor receptor [24]. SNX5 is expressed abundantly in the kidney [25]. Obese, hyperinsulinaemic, insulin-resistant Zucker rats [26] on normal salt diet have decreased renal SNX5 expression, relative to lean Zucker rats (Fengmin Li, unpublished data). We hypothesised that renal SNX5 regulates renal IDE and therefore circulating insulin levels and that renal-selective reduction of SNX5 may cause insulin resistance in vivo. Therefore, we studied the dynamic interaction between SNX5 and IDE in human RPTCs (hRPTCs), as well as in rat and mouse kidneys.

\section{Methods}

Cell lines Immortalised RPTCs (passage $<20$, mycoplasmafree) from humans and rats were provided with authorisation by R. A. Felder of the University of Virginia [21, 22]. The cells, randomly grouped for treatments (simple randomisation), were cultured at $37^{\circ} \mathrm{C}$ in $95 \%$ air and $5 \%$ $\mathrm{CO}_{2}$ (vol./vol.) in DMEM containing Nutrient Mixture F-12 (DMEM/F12) (Invitrogen, Life Technologies, Grand Island, NY, USA) supplemented with $10 \%$ (wt/vol.) fetal bovine serum (Sigma-Aldrich, St Louis, MO, USA), $1 \%$ (wt/vol.) penicillin-streptomycin (Invitrogen) and epidermal growth factor $(10 \mathrm{ng} / \mathrm{ml})$ (Sigma-Aldrich).

Animal studies Male C57B1/6J mice (1 year old) from Jackson Laboratory (Bar Harbor, ME, USA) were housed in standard facilities. Mice were uninephrectomised 3 weeks before a 7 day renal subcapsular infusion of small interfering (si)RNA ( $S N X 5$-specific or non-silencing 'mock' siRNA at $3 \mu \mathrm{g} /$ day, simple randomisation) into the remaining kidney, via an osmotic mini-pump (Alzet 1007D; Durect, Cupertino, CA, USA) $[21,22,27]$. The mice that were placed in metabolic cages for urine collection had free access to drinking water but food was withheld in some mice for $6-22 \mathrm{~h}$ prior to obtaining blood from the tail vein for measurement of glucose (AlphaTRAK 2 blood glucose monitoring system; Abbott, North Chicago, IL, USA) and insulin (ELISA; Crystal Chem, Downers Grove, IL, USA). The kidneys were harvested and homogenised for immunoblotting of SNX5 and IDE $[21,22]$. In additional studies in mice, insulin sensitivity/ 
resistance $[28,29]$ was tested by monitoring non-fasting blood glucose (AlphaTRAK 2) every $15 \mathrm{~min}$ for $60 \mathrm{~min}$, following insulin administration $(0.75 \mathrm{U} / \mathrm{kg}$, s.c. injection) on day 7 of the renal-selective siRNA infusion and day 21 (i.e. 14 days after stopping the renal-selective siRNA infusion). In additional studies in mice fasted for $6 \mathrm{~h}$, a glucose tolerance test was performed by monitoring the blood glucose level every $30 \mathrm{~min}$ for $120 \mathrm{~min}$ following glucose administration ( $1 \mathrm{~g} / \mathrm{kg}$ of body weight, i.p. injection) [28, 29]. The quantity of glucose in the blood (i.e. AUC [28, 29]), was calculated from 0 to $60 \mathrm{~min}$ in the insulin injection study and from 0 to $120 \mathrm{~min}$ in the glucose tolerance study.

Male Wistar-Kyoto (WKY) rats and spontaneously hypertensive rats (SHRs), 2 months old, from Japan SLC (Sendai, Japan), were housed in standard facilities. Eight of the WKY rats were uninephrectomised, as in the C57Bl/ 6 mice, and then given a 7 day renal cortical interstitial infusion of siRNA (Snx5-specific or mock siRNA at $3 \mu \mathrm{g} /$ day) into the remaining kidney, via an osmotic mini-pump [30]. Blood and kidney samples were collected after the rats were decapitated.

All animal studies were conducted in accordance with National Institutes of Health guidelines for the ethical treatment and handling of animals in research, and approved by the Institutional Animal Care and Use Committees of Georgetown University (Washington, DC, USA), University of Maryland (Baltimore, MD, USA) and Fukushima Medical University School of Medicine Animal Committee (Fukushima, Japan).

Western blotting Total cell lysates and kidney homogenates were prepared on ice using RIPA lysis buffer, supplemented with a cocktail of protease and phosphatase inhibitors (Thermo Fisher Scientific, Rockford, IL, USA). Equal amounts of protein $(30 \mu \mathrm{g})$ were electrophoresed on a $10 \%$ (wt/vol.) SDS-polyacrylamide gel and electro-transferred onto nitrocellulose membranes. The following primary antibodies (diluted 1:500) were used: goat anti-SNX5 (Santa Cruz Biotechnology, Dallas, TX, USA); mouse anti-IDE (GeneTex, Irvine, CA, USA); rabbit anti-cathepsin D (Cell Signaling Technology, Danvers, MA, USA); mouse antiglyceraldehyde 3-phosphate dehydrogenase (GAPDH, GeneTex) and rabbit anti-actin (Sigma-Aldrich). The specificity of the anti-SNX5 antibody was reported previously [31]. A rabbit polyclonal anti-IDE antibody (described below) was also used to confirm the specificity of the mouse IDE antibody. The secondary antibodies $(1: 10,000)$ were obtained from Santa Cruz Biotechnology or LI-COR Biosciences (Lincoln, NE, USA). The band densities of the proteins were quantified using either Image J software (NIH, Bethesda, MD, USA) or the Odyssey CLx Imaging System (LI-COR Biosciences) and expressed as a percentage of the relevant control density.
Immunofluorescence confocal microscopy hRPTCs were grown to $50-60 \%$ confluence on poly-D-lysine-coated coverslips and immunostained with goat polyclonal anti-SNX5 antibody (1:200; Santa Cruz Biotechnology), rabbit polyclonal anti-IDE antibody (1:200; Abcam, Cambridge, MA, USA) and then with the appropriate fluorophore-conjugated secondary antibodies (1:500; Molecular Probes, Grand Island, NY, USA).

Human kidney sections were obtained from a nonpathological section of a healthy kidney from a man (Imgenex/Novus Biologicals, Littleton, CO, USA). Kidney sections from WKY rats and $\mathrm{C} 57 \mathrm{Bl} / 6 \mathrm{~J}$ mice were also studied. The glass slides containing the kidney sections were incubated in xylene for $1 \mathrm{~h}$ in a dry oven at $60^{\circ} \mathrm{C}$ for deparaffinisation. The incubated glass slides were washed three times $(3 \mathrm{~min}$ each time) in $100 \%, 95 \%$ and $75 \%$ (vol./vol.) ethanol. The slides were boiled in sodium citrate buffer $(\mathrm{pH}$ 6.0), for $3 \mathrm{~min}$ for antigen retrieval and then incubated in $1 \%$ (wt/ vol.) bovine serum albumin blocking buffer for $30 \mathrm{~min}$ at room temperature. Double-staining with anti-SNX5 and antiIDE antibodies was performed. The primary antibodies were added onto the tissues and incubated at $4^{\circ} \mathrm{C}$ overnight. The secondary antibodies were subsequently added and incubated for $1 \mathrm{~h}$ at room temperature. The slides were mounted using ProLong Gold Antifade Mountant (Thermo Fisher Scientific) and imaged with a LSM 510 confocal microscope (Carl Zeiss, Oberkochen, Germany).

Co-immunoprecipitation Immunoprecipitation was performed using an Immunoprecipitation Kit (Protein G; Roche Applied Science, Indianapolis, IN, USA). Uniform amounts $(800 \mu \mathrm{g})$ of protein were immunoprecipitated with the primary antibody $(2 \mu \mathrm{g})$ specific for the protein of interest for $2 \mathrm{~h}$. Normal rat, mouse, and human IgGs (Santa Cruz Biotechnology) were used as negative controls. The samples were boiled for $5 \mathrm{~min}$ before western blotting.

RNA preparation and reverse transcriptase-quantitative real-time PCR For reverse transcriptase-quantitative realtime PCR (RT-qPCR), the following reactions were all performed in a C1000 Thermal Cycler machine (Bio-Rad, Hercules, CA, USA). Total RNA was extracted using an RNeasy plus mini kit (Qiagen Sciences, Germantown, MD, USA) and equal amounts of RNA (1 $\mu \mathrm{g})$ were loaded for cDNA synthesis using a Tetro cDNA Synthesis Kit (Bioline USA, Taunton, MA, USA). RT-qPCR was then performed, using SYBR Green PCR Master Mix (Qiagen Sciences) in a 7900HT Fast Real-Time PCR machine (Applied Biosystems, Life Technologies, Grand Island, NY, USA). Relative quantities of SNX 5 and IDE were normalised by $\beta$-actin. Thermal cycler settings were as follows: $50^{\circ} \mathrm{C}$ for $2 \mathrm{~min}, 95^{\circ} \mathrm{C}$ for $10 \mathrm{~min}, 40$ cycles of $95^{\circ} \mathrm{C}$ for $15 \mathrm{~s}, 59^{\circ} \mathrm{C}$ for $1 \mathrm{~min}, 95^{\circ} \mathrm{C}$ for $15 \mathrm{~s}$ and $60^{\circ} \mathrm{C}$ for $15 \mathrm{~s}$. 
The gene-specific primer pairs were as follows: SNX5 forward (5'-ACGTTTCAGAGCCCAGAGTT-3') and SNX5 reverse (5'-TCGAGGACCATCAAAGTCG-3'); IDE forward (5'-TTCCAAGAAGAACATCTTAAACAACT-3') and IDE reverse (5'-ACCTTCATGCCCAATGAGAT-3'); $\beta$-actin forward (5'-ACCTGTACGCCAACACAGTG-3') and $\beta$-actin reverse (5'-ACACGGAGTACTTGCGCTCA-3').

\section{SNX5-specific siRNA/small hairpin RNA transfection} Lipofectamine RNAiMAX transfection reagent (Thermo Fisher Scientific) was used to transfect SNX5-specific siRNA (Santa Cruz Biotechnology) into hRPTCs. The cells were seeded into six-well plates, 1 day before the transfection. Serum-free medium was used once the cells reached $70-80 \%$ confluence. The cells were incubated in a mixture of $4 \mu \mathrm{l}$ transfection reagent and $5 \mu \mathrm{l}$ siRNA stock solution (10 $\mu \mathrm{mol} / \mathrm{l})$ at room temperature for 20-30 min. At about $6 \mathrm{~h}$ post-transfection, the same amount of complete medium was added into each well. The cells were harvested after $48 \mathrm{~h}$ for RNA quantification using RT-qPCR and after $72 \mathrm{~h}$ for total protein quantification by western blot.

FuGENE 6 transfection reagent (Promega, Madison, WI, USA) was used to transfect $S N X 5$ small hairpin RNA (shRNA) into hRPTCs. The clones were isolated by adding puromycin 1 day after transfection and incubating for 14 days at $37^{\circ} \mathrm{C}$. We tested several shRNAs for their ability to silence the expression of $S N X 5$ and used the shRNA that gave the greatest downregulation of $S N X 5$ expression.

IDE activity InnoZyme Insulysin/IDE Immunocapture Activity Assay Kit (Calbiochem, EMD Millipore, Billerica, MA, USA) was used to measure IDE activity. hRPTCs were lysed with CytoBuster Protein Extraction Reagent (EMD Millipore).

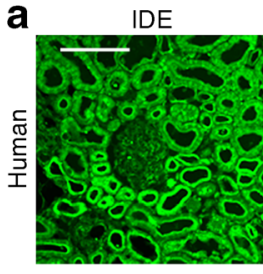

IDE

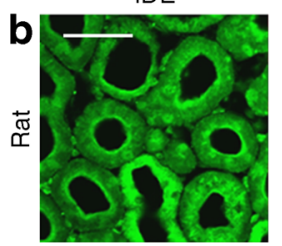

Fig. 1 Co-localisation of IDE and SNX5 in the kidney. Paraffin-embedded human (a) and rat (b) kidney sections were double-immunostained for endogenous IDE (pseudocoloured green) and SNX5 (pseudocoloured red), after antigen retrieval. Co-localisation of IDE and SNX5 (merge) is visualised as discrete yellow areas mainly located at the
Fluorescence energy transfer microscopy and data processing The fluorophore pairs (Invitrogen) used for fluorescence energy transfer (FRET) imaging were Alexa Fluor 555 (acceptor dipole) conjugated with SNX5 antibody and Alexa Fluor 488 (donor dipole) conjugated with IDE. Seven images were acquired for each FRET analysis, using an Olympus FluoView FV300 confocal laser scanning microscope (Olympus Corporation of the Americas, Center Valley, PA, USA) equipped with a $60 \times / 1.4$ NA objective, Argon $(488 \mathrm{~nm}), \mathrm{HeNe}(543 \mathrm{~nm})$ laser, emission filters of 515/ $50 \mathrm{~nm}$ and a $590 \mathrm{~nm}$ long pass filter.

Statistical analysis All experimenters were blind to group assignment and outcome assessment. We included all outcomes for data report and analysis. Numerical data are reported as means \pm SEM. Significant difference between two groups was determined by Student's $t$ test. Significant differences among groups were determined by one-way factorial ANOVA, followed by Student-Newman-Keuls post hoc test. $p<0.05$ was considered significant.

\section{Results}

IDE and SNX5 co-localise in several nephron segments in the kidney We have reported the presence of SNX5 in different segments of the nephron, including the proximal tubule, in human and rat kidneys [21]. We now show that IDE and SNX5 co-localise in human and rat kidneys, mainly at the brush border membrane of proximal tubules and luminal side of distal convoluted tubules (Fig. 1).

IDE and SNX5 dynamically interact in renal proximal tubules In cultured hRPTCs from normotensive volunteers, IDE and SNX5 were basally expressed to a lesser extent in the

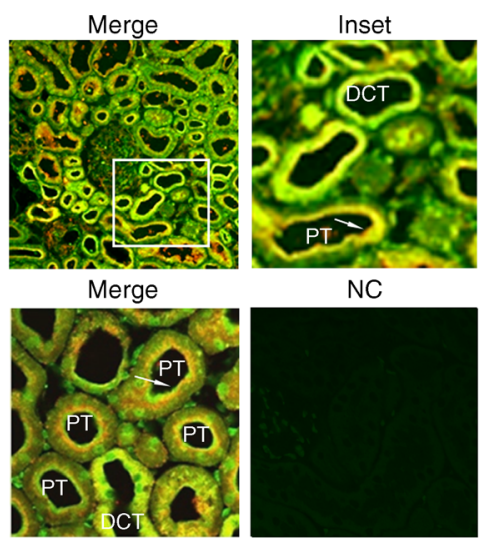

brush border membrane (indicated by arrow) of proximal tubules (PT), characterised by thick walls and brush border membrane, and at the luminal side of distal convoluted tubules (DCT), characterised by thin walls and spacious lumens. NC, negative control without primary antibodies. Scale bars: (a) $40 \mu \mathrm{m}$ and (b) $20 \mu \mathrm{m}$ 
plasma membrane and a greater extent in the cytoplasm, especially in the perinuclear area, although the co-localisation was minimal. Insulin treatment ( $100 \mathrm{nmol} / 1,30 \mathrm{~min}$ ) increased the co-localisation of IDE and SNX5 at the perinuclear area and plasma membrane (Fig. 2a). At $30 \mathrm{~min}$, the FRET between IDE and SNX5 had an energy transfer efficiency around $50 \%$ (Fig. 2b).

The physical interaction of IDE and SNX5 was confirmed by co-immunoprecipitation. Co-immunoprecipitation of SNX5 and IDE was negative in the basal state $(0 \mathrm{~min})$ but occurred after insulin treatment (100 nmol/1, $30 \mathrm{~min})$ (Fig. 2c).

SNX5 silencing decreases IDE expression and activity shRNA-mediated silencing of SNX5 [31] caused a marked reduction in the expression of SNX5 protein (Fig. 3a) and
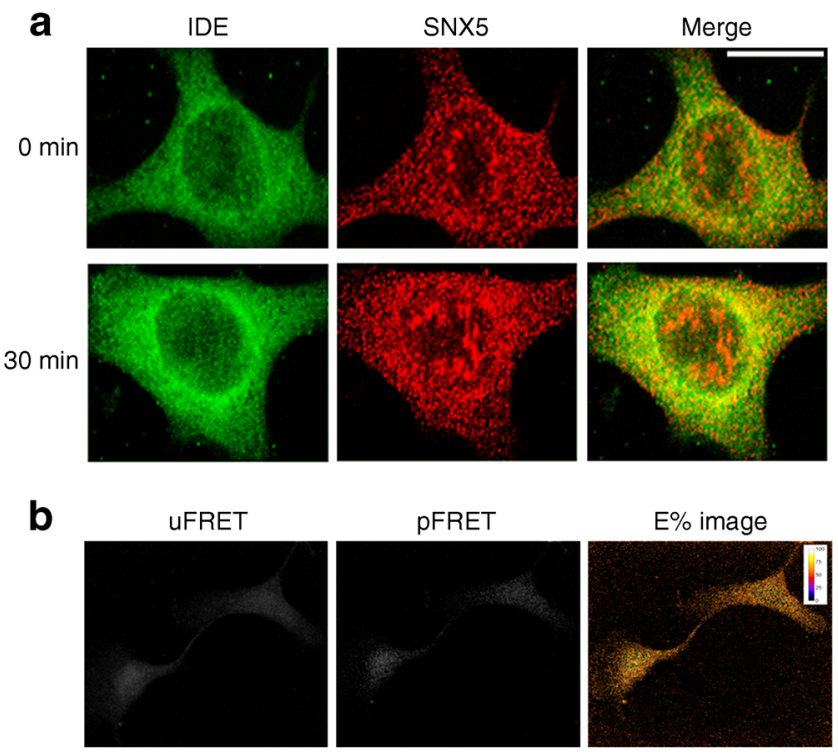

b

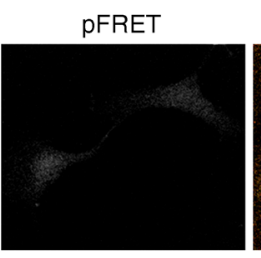

E\% image

C

IP: SNX5
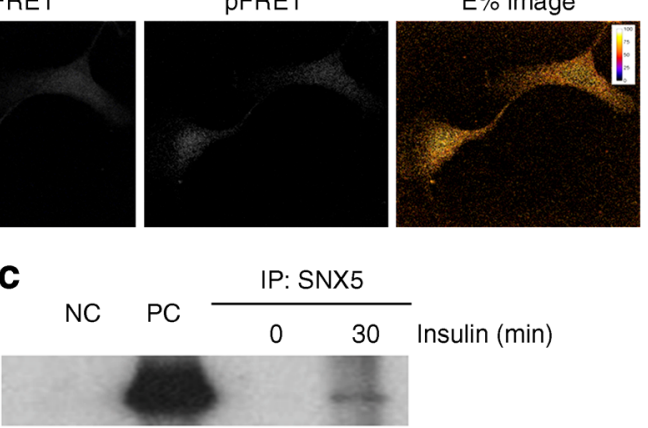

Fig. 2 Co-localisation and co-immunoprecipitation of IDE and SNX5 in hRPTCs. (a) Co-localisation of IDE and SNX5 in hRPTCs from normotensive human volunteers. The cells were washed, fixed and immunostained for IDE and SNX5. Co-localisation is visualised as yellow discrete areas in merged images of IDE (pseudocoloured green) and SNX5 (pseudocoloured red). There is co-localisation at the plasma membrane and perinuclear area in the basal state. Insulin treatment $(100 \mathrm{nmol} /$ 1, $30 \mathrm{~min}$ ) increased the co-localisation at the plasma membrane and perinuclear area; scale bar, $5 \mu \mathrm{m}$. (b). FRET between IDE and SNX5 in hRPTCs. Alexa Fluor 488-labelled IDE was used as the donor and Alexa Fluor 555-labelled SNX5 was used as the acceptor in the FRET dipole. At $30 \mathrm{~min}$, the FRET between IDE and SNX5 had an energy transfer efficiency (E\%) around 50\%. uFRET, uncorrected FRET; pFRET, processed FRET. (c) Co-immunoprecipitation of IDE and SNX5 in hRPTCs. The cells were immunoprecipitated (IP) with SNX5 antibodies and immunoblotted for IDE. The co-immunoprecipitation of IDE and SNX5 occurred following insulin treatment (100 nmol/1, $30 \mathrm{~min})$. NC, negative control with non-specific IgG; PC, positive control with regular immunoblotting for IDE of the cell lysates modest reduction of IDE protein (Fig. 3b) in hRPTCs. Moreover, these $S N X 5$-silenced cells had decreased IDE activity (Fig. 3c). Similarly, siRNA-mediated silencing of SNX5 resulted in a marked reduction in the expression of SNX5 protein (Fig. 3d) and SNX5 mRNA $(1.0 \pm 0.06 \mathrm{vs}$ $0.32 \pm 0.02, p<0.05, n=3$ /group, fold change over control). SNX5 siRNA, as with $S N X 5$ shRNA, also slightly decreased $I D E$ mRNA expression (Fig. 3e). There was specificity of the effect of SNX5 on IDE expression because the expression of cathepsin D protein, an aspartic protease that has insulinase activity at pH 5 in endosomes [15], was not significantly altered in SNX5-depleted hRPTCs (Fig. 3f).

Silencing of renal SNX5 decreases renal IDE in mice IDE and SNX5 co-localised in mouse renal cortex, mainly at the brush border membrane of proximal tubules (Fig. 4a), as in human and rat kidneys (Fig. 1). We next selectively silenced the mouse renal $\operatorname{Snx} 5$ (Fig. 4b) via renal subscapular infusion $[21,22,27]$ of $\operatorname{Snx} 5$-specific siRNA to determine the in vivo correlates of our observations in cells. We found that renalselective silencing of Snx 5 (SNX5 protein: $100 \pm 25$ vs $29 \pm 10, p<0.05)$ (Fig. 4b) decreased IDE protein expression (Fig. 4 c) $(100 \pm 13$ vs $57 \pm 6, p<0.05)$. The reduction in SNX5 and IDE expression was also confirmed by immunostaining (electronic supplementary material [ESM] Fig. 1). The reduction in SNX5 and IDE expression was not accompanied by a change in renal structure (ESM Fig. 2).

Renal-selective silencing of $\operatorname{Sn} x 5$ increases blood insulin and glucose levels, decreases urinary insulin excretion, and causes insulin resistance in mice In renal Snx5-depleted mice, fasting serum insulin was increased and urinary insulin excretion was decreased (Fig. 5a, b). In another set of mice, we quantified the non-fasting blood glucose levels in response to a subcutaneous injection of insulin on day 7 of the renal subcapsular infusion of Snx5 siRNA. Snx 5 siRNA-treated mice, relative to mock siRNA-treated mice, had increased blood glucose levels before the injection of insulin. In Snx5 siRNA-treated mice, insulin decreased the blood glucose to levels seen in mock siRNA-treated mice at $15 \mathrm{~min}$ but blood glucose levels started to increase and became significantly higher than those in mock siRNA-treated mice at 45 and 60 min (Fig. 5c). The blood glucose of mock siRNA-treated mice remained at the same low levels from 15 to $60 \mathrm{~min}$ (Fig. 5c); the AUC from 0 to 60 min was higher in Snx 5 siRNA-treated mice than in mock siRNA-treated mice (Fig. 5d). On day 21 (14 days after stopping the siRNA infusion), blood glucose levels before and after insulin were no longer different between the two groups of mice (ESM Fig. 3a,b). In additional studies in mice, on day 7 of siRNA infusion, the $6 \mathrm{~h}$ fasting blood glucose levels were similar at baseline, increased by the same extent 15 min after a glucose load but remained higher over the next $30 \mathrm{~min}$ in Snx 5 siRNA-treated mice than in mock siRNA-treated mice 


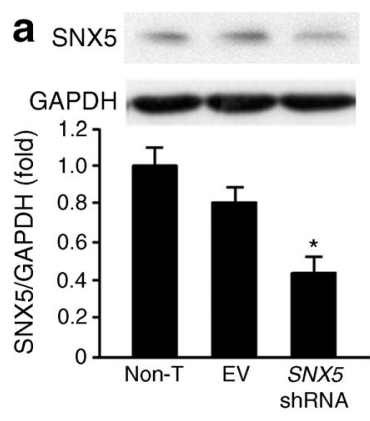

d

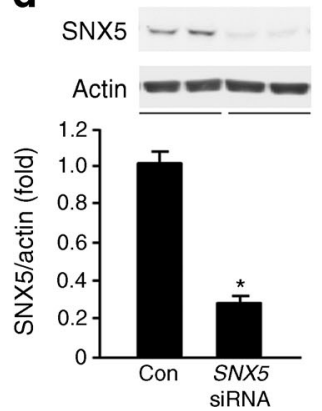

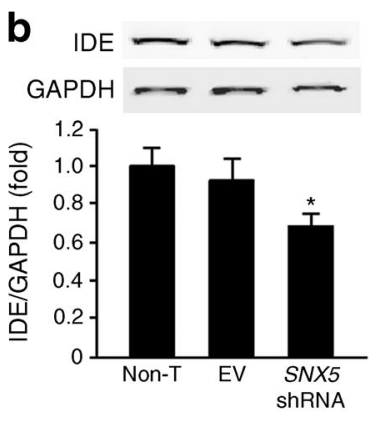

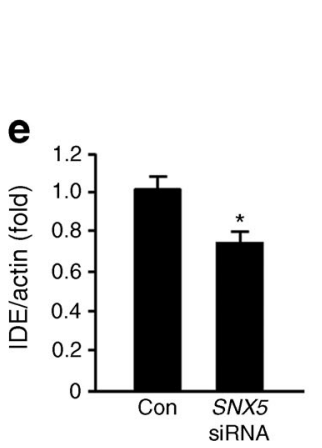

C

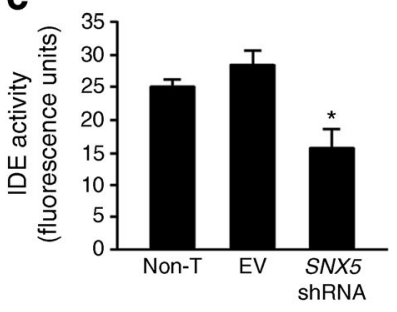

f Cathespsin D

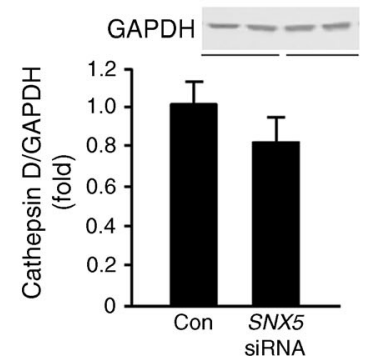

Fig. 3 IDE expression and activity in SNX5-depleted hRPTCs. (a-c). SNX5 protein expression (a) and IDE protein expression (b) (shown as fold change) and activity (c) (shown as relative fluorescence units) were decreased in SNX5 shRNA-transfected hRPTCs, compared with nontransfected (Non-T) and empty vector-transfected (EV) hRPTCs. $n=3$ / group; $* p<0.05$ vs Non-T and EV (one-way ANOVA, StudentNewman-Keuls test). Data were normalised by GAPDH $(\mathbf{a}, \mathbf{b})$ or by protein concentration (c). (d) SNX5 protein was decreased by SNX5 siRNA treatment. $n=6 /$ group; ${ }^{*} p<0.05$ vs control (Con) (Student's $t$

(Fig. 5e). The blood glucose AUC from 0 to 120 min was increased by $50 \%$ in the $\operatorname{Snx} 5$ siRNA-treated mice relative to the mock siRNA-treated mice (Fig. 5f).

\section{Relationship between hypertension and insulin resistance} The SHR is not only genetically hypertensive but also has increased fasting serum levels of insulin and is insulin resistant $[32,33]$. Therefore, we measured renal SNX5 and IDE expression and serum insulin and glucose levels in SHRs and their controls, WKY rats. We found that renal SNX5 (Fig. 6a) $(100 \pm 27 \%$ vs $29 \pm 6 \%, p<0.05)$ and IDE (Fig. 6b) $(100 \pm 5 \%$ vs $75 \pm 4 \%, p<0.05)$ proteins were decreased, while non-fasting serum insulin and glucose levels (Fig. 6c, d) were increased in SHRs, relative to WKY rats. Increased nonfasting serum insulin and glucose levels (Fig. 6e, f) were also found in WKY rats selectively depleted of renal SNX5 [21]. These findings demonstrate an association between decreased renal SNX5 and IDE expression and increased serum insulin and glucose levels.

We also evaluated the expression of SNX5 and IDE in immortalised RPTCs from WKY rats and SHRs, as well as in immortalised RPTCs from hypertensive and normotensive humans. We found that expression of SNX5 and IDE in test). Data were normalised by actin and expressed as fold change. (e) $I D E$ mRNA was decreased by SNX5 siRNA treatment. $n=6 /$ group; $* p<0.05$ vs Con (Student's $t$ test). Data were normalised by actin and expressed as fold change. (f) Cathepsin D protein expression in SNX5 siRNA-transfected hRPTCs. Expression of cathepsin D, another insulinase, was not significantly changed in SNX5 siRNA-transfected hRPTCs, relative to Con. $n=3 /$ group; $p>0.05$ (Student's $t$ test). Data were normalised by GAPDH and expressed as fold change

RPTCs was decreased in SHRs (Fig. 7a, b) and hypertensive humans (Fig. 7c, d), relative to WKY rats and normotensive humans, respectively.

\section{Discussion}

SNX5, a protein composed of 404 amino acids, contains a central phox homology (PX) domain and large C-terminal domain predicted to include a BAR domain [34]. SNX5 is expressed in many organs, with the highest levels of SNX5 mRNA being detected in skeletal muscle and kidney [25]. Since first being identified by its interaction with the Fanconi anaemia complementation group A protein [25], SNX5 has been implicated in a myriad of cellular processes, such as trafficking and degradation of $G$ protein-coupled and epithelial growth factor receptors [19-21, 24, 31]. Some studies have shown that SNX5 is associated with several diseases, including hypertension [21]. We have reported that renal SNX5, along with $D_{1} R$, is required for the normal expression of the insulin receptor in the kidney [31]. We now report the biological role of renal SNX5 in insulin metabolism. 


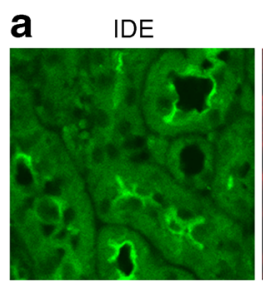

b

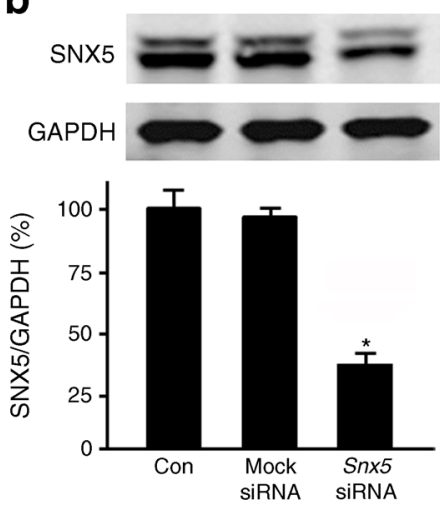

Fig. 4 Renal Snx5 silencing in mice. (a) Co-localisation of SNX5 and IDE in normal mouse kidney cortex. Paraffin-embedded sections were co-immunostained for IDE (pseudocoloured green) and SNX5 (pseudocoloured red). Co-localisation (merge) is visualised as discrete yellow areas mainly at the brush border membrane of proximal tubules (blue arrows). DIC, differential interference contrast. Scale bar, $20 \mu \mathrm{m}$. (b) SNX5 protein expression in homogenates of mouse renal cortex was decreased in Snx5 siRNA-treated kidneys, relative to control (Con) and

The kidney is the major organ responsible for the clearance of insulin from the systemic circulation [11-13]. Individuals with chronic renal failure have increased plasma insulin levels [35]. The ability of the kidney to clear insulin is due to the presence of IDE [12] but the mechanism by which this occurs is not well understood. The current study shows for the first time a novel mechanism by which IDE is regulated in the kidney. We found that SNX5 dynamically interacts with IDE in hRPTCs and that silencing SNX5 in these cells decreases the expression and activity of IDE. The decrease in activity is probably due to a decrease in the protein expression, since enzyme activity was measured with saturating concentrations of substrate, which reflect the total amount of enzyme [36]. The decreased IDE protein expression with SNX5 depletion was associated with decreased IDE mRNA expression, indicating that the regulation may be at the transcription level, although the ability of SNX5 to promote IDE degradation cannot be ruled out. The abundance of another insulinase, cathepsin D [15], was unaltered.

The positive regulation of renal IDE activity by SNX5 has physiological consequences. We now report that SNX5 also affects circulating insulin levels through its ability to regulate renal IDE, the enzyme that is primarily responsible for insulin degradation $[5-8,15-17,36]$. SNX5 is required for normal IDE expression and function in the kidney. The absence of
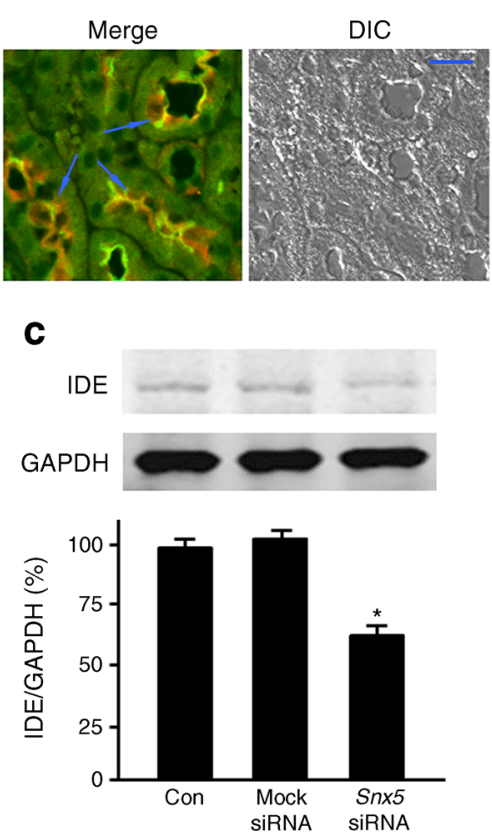

mock siRNA (Mock) groups. $n=4$ /group; $* p<0.05$ vs Con and Mock (one-way ANOVA, Student-Newman-Keuls test). Data were normalised by GAPDH. (c) IDE protein expression in homogenates of mouse renal cortex was decreased in Snx 5 siRNA-treated kidneys, relative to Con and Mock groups. $n=4 /$ group; $* p<0.05$ vs Con and Mock (one-way ANOVA, Student-Newman-Keuls test). Data were normalised by GAPDH

SNX5 results in decreased abundance and activity of IDE, leading to decreased urinary insulin excretion and increased circulating insulin levels, even in the fasting state. Under normal circumstances, the increased insulin levels should promote the use of glucose by peripheral tissues and, thus, should decrease serum glucose levels. However, the non-fasting glucose level was increased in mice 7 days after renal-selective depletion of SNX5 by the renal subcapsular infusion of Snx5specific siRNA. The blood glucose levels, 45-60 min after insulin injection, were higher in SNX5-depleted mice than in control mice but become normal 14 days after stopping the siRNA infusion. The renal-selective depletion of SNX5 also impaired the response to a glucose load. These data demonstrate the occurrence of insulin resistance in mice with decreased renal SNX5. We have previously reported that kidney-restricted silencing of Snx5 in mice results in decreased expression of the insulin receptor and decreased abundance of phosphorylated insulin receptor substrate and protein kinase B [31]. It is conceivable that a compensatory increase in the secretion of insulin from pancreatic beta cells may also contribute to the increase in circulating insulin in renal SNX5deficient mice [37]. Indeed, the initial (15 min) increase in blood glucose in response to the glucose load was similar in SNX5-depleted and control mice. However, the decrease in urinary insulin excretion in SNX5-depleted mice suggests that 
Fig. 5 Serum insulin, urinary insulin excretion and blood glucose response to insulin and glucose in mice with Snx5 siRNA-treated kidneys. (a) Fasting serum insulin was increased in mice with Snx5 siRNA-treated kidneys. $n=4 /$ group; ${ }^{*} p<0.05$ vs mock siRNA (Mock) treatment (Student's $t$ test). (b) Fasting urinary insulin excretion was decreased in mice with $S n x 5$ siRNA-treated kidneys. $n=4$ /group; ${ }^{*} p<0.05$ vs Mock (Student's $t$ test). Data were normalised by urine creatinine $(\mathrm{Cr})$. (c) Non-fasting blood glucose levels in response to insulin on day 7 of renal-selective Snx 5 siRNA infusion. Non-fasting blood insulin was higher in mice treated with $\operatorname{Snx} 5$ siRNA (squares, dashed line) than in Mock-treated mice (triangles, solid line) before the s.c. injection of insulin $(0.75 \mathrm{U} / \mathrm{kg})$. Insulin injection decreased blood glucose to the same level in Snx5 siRNA- and Mock-treated mice at $15 \mathrm{~min}$ but blood glucose levels started to increase and became significantly higher in Snx5 siRNA- than in Mock-treated mice at 45 and $60 \mathrm{~min}$; blood glucose levels of Mock-treated mice remained at the same low levels from 15 to $60 \mathrm{~min}$. $n=4$ /group; * $p<0.05$ vs Mock (Student's $t$ test). (d) AUC of non-fasting blood glucose levels in response to insulin on day 7 of renal-selective $S n x 5$ siRNA or Mock siRNA infusion. AUC was calculated as $\mathrm{mmol} / \mathrm{l} \times 60 \mathrm{~min}$ and expressed as a percentage of the AUC obtained with Mock treatment. Snx5 siRNAtreated mice had increased blood glucose AUC, relative to Mocktreated mice. $n=4 /$ group $* p<0.05$ vs Mock (Student's $t$ test). (e) Fasting blood glucose levels in response to an i.p. glucose load $(1 \mathrm{~g} / \mathrm{kg}$ of body weight) on day 7 of renal-selective $S n x 5$ siRNA or Mock siRNA infusion. The blood glucose was increased to the same level in Snx5 siRNA-treated mice (squares, dashed line, $n=4$ ) and Mock-treated mice (triangles, solid line, $n=5$ ) at $15 \mathrm{~min}$ but remained higher in $S n x 5$ siRNA-treated mice than in Mock-treated mice at 30 and $60 \mathrm{~min}$; ${ }^{*} p<0.05$ vs Mock (Student's $t$ test). (f) AUC of fasting blood glucose levels in response to the i.p. glucose load on day 7 of renal-selective $\operatorname{Snx} 5$ siRNA or Mock siRNA infusion. AUC was calculated as $\mathrm{mmol} / \mathrm{l} \times 120 \mathrm{~min}$ and expressed as a percentage of the AUC obtained with Mock siRNA treatment. Snx5 siRNA-treated mice had increased blood glucose AUC, relative to Mock-treated mice. $n=4$ or 5/group; $* p<0.05$ vs Mock (Student's $t$ test)

renal SNX5 plays a major role in insulin resistance through the positive regulation of renal IDE (current study) and insulin receptors [31].

Another finding in this study is that RPTCs from hypertensive rats and humans have markedly lower renal SNX5 and IDE expression, relative to their respective normotensive controls (i.e. WKY rats and healthy humans). SHRs had lower renal SNX5 and IDE but had higher serum insulin and glucose levels than WKY rats. Similarly, renal SNX5-depleted WKY rats also had increased serum insulin and glucose levels. Thus, our results demonstrate a novel mechanism for the development of both hypertension [21] and insulin resistance (current study) (i.e. decreased renal SNX5).

We have previously ascribed the hypertension that develops in SNX5-deficient mice to the consequent impairment of renal $\mathrm{D}_{1} \mathrm{R}$ activity [21]. We now propose hyperinsulinaemia as a contributing mechanism for the elevation of blood pressure in these mice. Insulin acts on all the nephron segments but accumulates to the greatest degree in the proximal tubule [38] to increase sodium reabsorption. Insulin has contrasting effects on various mechanisms that influence blood pressure. On the one hand, it promotes sodium retention and increases sympathetic activity

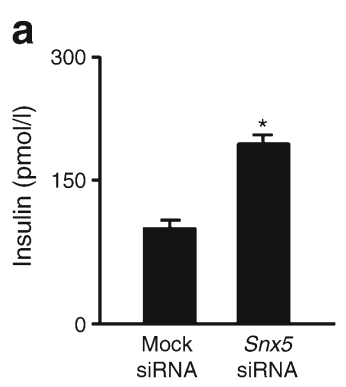

b
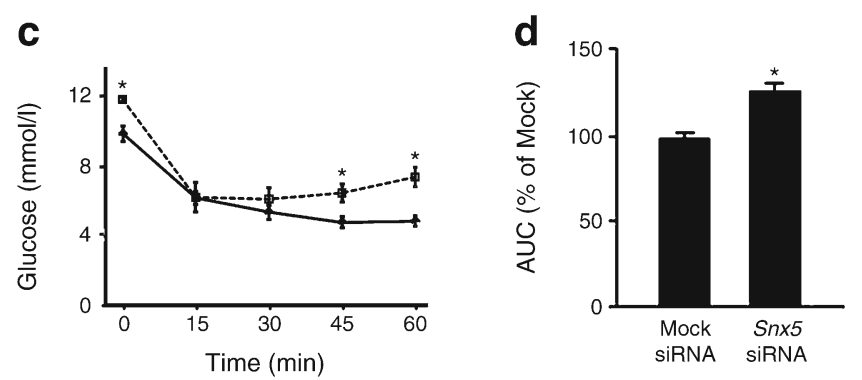

e

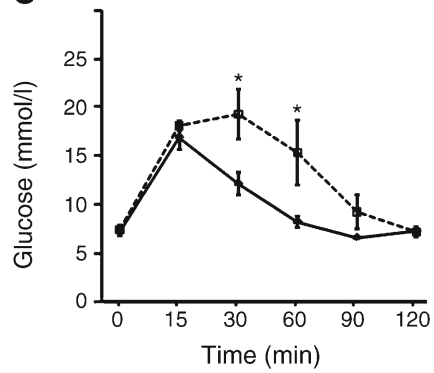

f

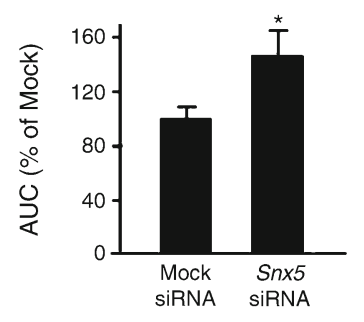

[39] while on the other, insulin, per se, has direct vasodilatory effects [40] and chronic insulin infusion into animals does not significantly increase blood pressure [41]. However, the effect of insulin on blood pressure may be altered in the presence of insulin resistance, as is the case in our mice. Insulin-mediated vasodilatation may be impaired due to defects in PI3-kinase signalling [42], while insulin-mediated sodium retention may be preserved or even enhanced [43, 44] in insulin resistance; both leading to a heighted effect of hyperinsulinaemia on blood pressure. These effects may take place despite the seeming decrease in renal insulin receptor levels in SNX5-deficient mice.

Taken together, we suggest that SNX5 is pivotal in insulin and glucose metabolism and that loss or deficiency of SNX5 results in the development of insulin resistance through absence or reduced SNX5 regulation of renal insulin receptors and IDE (Fig. 8). Although an SNX5 variant (rs6045116) was not associated with salt-sensitive hypertension in Americans or those of European descent [45], it is conceivable that other gene variants may potentially affect the expression, localisation and activity of SNX5. The SNX5 gene is replete with SNPs, such as rs754299876, rs758576026 and rs746381456, and structural variations, such as changes in copy number and indels (insertions and deletions), that may associate with or link to hypertension, salt sensitivity and 

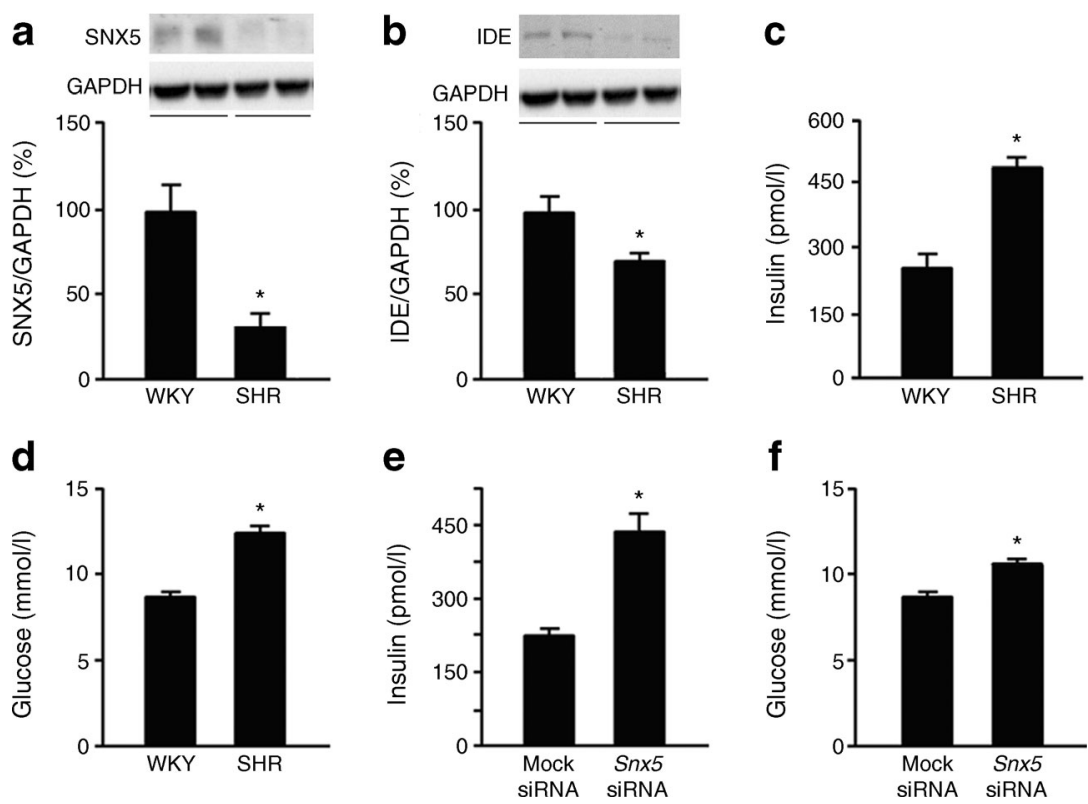

Fig. 6 Renal SNX5, IDE, and serum insulin and glucose levels in WKY rats and SHRs. (a) Renal expression of SNX5 was decreased in SHRs, relative to WKY rats. $n=3$ /group; $* p<0.05$ vs WKY rats (Student's $t$ test). Data were normalised by GAPDH and expressed in $\%$. (b) Renal expression of IDE was decreased in SHRs $(n=7)$, relative to WKY rats $(n=8), * p<0.05$ vs WKY rats (Student's $t$ test). Data were normalised by GAPDH and expressed in \%. (c) Non-fasting serum insulin level was increased in SHRs, relative to WKY rats. $n=4$ /group;
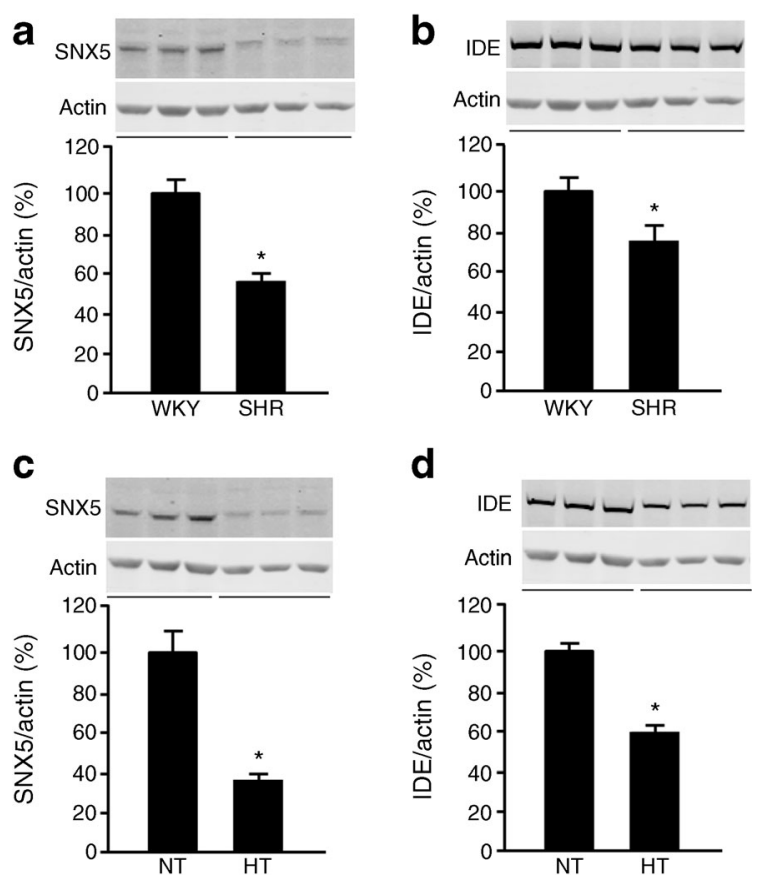

Fig. 7 SNX5 and IDE expression in RPTCs from rats and humans. $(\mathbf{a}, \mathbf{b})$ SNX5 (a) and IDE (b) protein expression was decreased in RPTCs from SHRs, relative to expression in RPTCs from WKY rats. $n=3$ /group; $* p<0.05$ vs WKY (Student's $t$ test). Data were normalised by actin and expressed in \%. (c, d) SNX5 (c) and IDE (d) protein expression was decreased in RPTCs from hypertensive volunteers (HT, $n=3$ ), relative to expression in RPTCs from normotensive volunteers (NT, $n=6$ ), ${ }^{*} p<0.05$ vs NT (Student's $t$ test). Data were normalised by actin and expressed in $\%$
${ }^{*} p<0.05$ vs WKY rats (Student's $t$ test). (d) Non-fasting serum glucose was increased in SHRs, relative to WKY rats. $n=4$ /group; ${ }^{*} p<0.05$ vs WKY rats (Student's $t$ test). (e) Non-fasting serum insulin was increased in WKY rats with $S n x 5$ siRNA-treated kidneys, relative to WKY rats with mock siRNA-treated (Mock) kidneys. $n=4$ /group; $* p<0.05$ vs Mock (Student's $t$ test). (f) Non-fasting serum glucose was increased in WKY rats with $S n x 5$ siRNA-treated kidneys $(n=4)$, relative to WKY rats with Mock-treated kidneys $(n=5), * p<0.05$ vs Mock (Student's $t$ test)

insulin resistance. These relationships could be the subject of future studies. Furthermore, studies on the role of SNX5 in energy metabolism could indicate a yet undescribed role in the aetiology of metabolic syndrome, since hypertension and

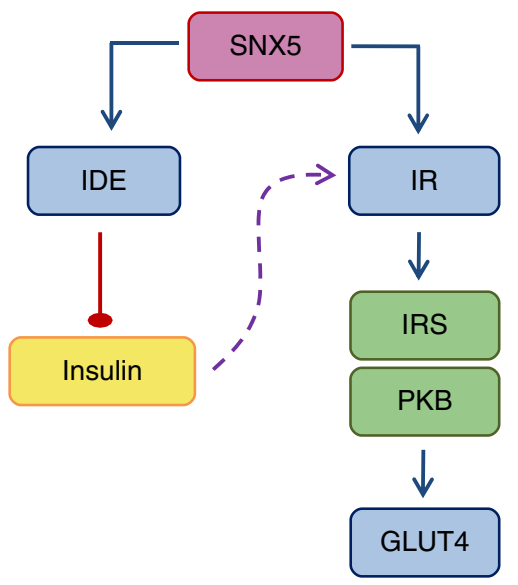

Fig. 8 The role of SNX5 in insulin and glucose metabolism. SNX5 is required for the normal expression and activity of both the IDE and insulin receptor (IR). Insulin degradation mainly results from activity of IDE in the kidney, where most of the circulating insulin is cleared. Insulin action starts with IR activation and signal transduction, which includes an increase in phosphorylation of IRS and protein kinase B (PKB), and terminates with the activation of GLUT4, which increases cellular glucose uptake. Loss or deficiency of renal SNX5 has the following effects: (1) decreased expression and activity of IDE, which leads to increased circulating insulin levels and (2) decreased IR expression, which leads to the development of insulin resistance 
insulin resistance are cornerstones of the syndrome. The human SNX5 gene maps at 20p11.23, a locus that has been implicated in the aetiology of the MOMO (macrosomia, obesity, macrocephaly and ocular abnormalities) syndrome [46], which includes obesity, another hallmark of the metabolic syndrome [47].

Data availability The data in this report are available on request to the corresponding author.

Funding These studies were supported, in part, by grants R37HL023081, P01HL068686, P01HL074940, R01HL092196, R01DK039308, and R01DK090918 from the US National Institutes of Health. XW was also supported by a Professional Development Award from the National Kidney Foundation of Maryland (NKF-MD) and Diabetes Action Research and Education Foundation. VMV was supported by a minigrant from NKF-MD and JY was also supported by grants 81100500 and 81570379 from the National Natural Science Foundation of China.

Duality of interest The authors declare that there is no duality of interest associated with this manuscript.

Contribution statement FL, JY, and VMV participated in the study conception and design and data acquisition and analyses of the cell experiments, and drafted, revised and approved the manuscript. LDA, XM, and IA participated in data acquisition and analyses for the mouse experiments and revised and approved the manuscript. HS and MY participated in the study conception and design, data acquisition and analyses for the rat experiments and revised and approved the manuscript. RAF participated in the conception and design of the cell experiments and revised and approved the manuscript. PAJ participated in the conception and design of all the experiments and revised and approved the manuscript. XW participated in the study conception and design and the data acquisition and analyses for the mouse and rat experiments and revised and approved the manuscript. XW and VMV are the guarantors of this work and, as such, had full access to all the data in the study. They take responsibility for the integrity of the data and the accuracy of the data analysis.

\section{References}

1. Tsatsoulis A, Mantzaris MD, Bellou S, Andrikoula M (2013) Insulin resistance: an adaptive mechanism becomes maladaptive in the current environment-an evolutionary perspective. Metabolism 62:622-633

2. Vieweg WV, Levy JR, Fredrickson SK et al (2008) Psychotropic drug considerations in depressed patients with metabolic disturbances. Am J Med 121:647-655

3. Bates GW Jr, Propst AM (2012) Polycystic ovarian syndrome management options. Obstet Gynecol Clin N Am 39:495-506

4. Shanik MH, Xu Y, Skrha J et al (2008) Insulin resistance and hyperinsulinemia: is hyperinsulinemia the cart or the horse? Diabetes Care 31(Suppl 2):S262-S268

5. Fakhrai-Rad H, Nikoshkov A, Kamel A et al (2000) Insulindegrading enzyme identified as a candidate diabetes susceptibility gene in GK rats. Hum Mol Genet 9:2149-2158

6. Karamohamed S, Demissie S, Volcjak J et al (2003) Polymorphisms in the insulin-degrading enzyme gene are associated with type 2 diabetes in men from the NHLBI Framingham Heart Study. Diabetes 52:1562-1567
7. Furukawa Y, Shimada T, Furuta H et al (2008) Polymorphisms in the IDE-KIF11-HHEX gene locus are reproducibly associated with type 2 diabetes in a Japanese population. J Clin Endocrinol Metab 93:310-314

8. Rudovich N, Pivovarova O, Fisher E et al (2009) Polymorphisms within insulin-degrading enzyme (IDE) gene determine insulin metabolism and risk of type 2 diabetes. J Mol Med (Berl) 87:11451151

9. Fawcett J, Sang H, Permana PA, Levy JL, Duckworth WC (2010) Insulin metabolism in human adipocytes from subcutaneous and visceral depots. Biochem Biophys Res Commun 402:762-766

10. Castell-Auví A, Cedó L, Pallarès V, Blay M, Ardévol A, Pinent M (2012) The effects of a cafeteria diet on insulin production and clearance in rats. Br J Nutr 108:1155-1162

11. Rabkin R, Ryan MP, Duckworth WC (1984) The renal metabolism of insulin. Diabetologia 27:351-357

12. Valera Mora ME, Scarfone A, Calvani M, Greco AV, Mingrone G (2003) Insulin clearance in obesity. J Am Coll Nutr 22:487-493

13. Hjelle JT, Oparil S, Peterson DR (1984) Subcellular sites of insulin hydrolysis in renal proximal tubules. Am J Phys 246:F409-F416

14. Fawcett J, Rabkin R (1993) Degradation of insulin by isolated rat renal cortical endosomes. Endocrinology 133:1539-1547

15. Authier F, Posner BI, Bergeron JJ (1996) Insulin-degrading enzyme. Clin Invest Med 19:149-160

16. Tundo GR, Sbardella D, Ciaccio C et al (2013) Insulin-degrading enzyme (IDE): a novel heat shock-like protein. J Biol Chem 288: 2281-2289

17. Farris W, Mansourian S, Chang Y et al (2003) Insulin-degrading enzyme regulates the levels of insulin, amyloid beta-protein, and the beta-amyloid precursor protein intracellular domain in vivo. Proc Natl Acad Sci U S A 100:4162-4167

18. Zhao L, Teter B, Morihara T et al (2004) Insulin-degrading enzyme as a downstream target of insulin receptor signaling cascade: implications for Alzheimer's disease intervention. J Neurosci 24:11120 11126

19. Cullen PJ, Korswagen HC (2011) Sorting nexins provide diversity for retromer-dependent trafficking events. Nat Cell Biol 14:29-37

20. Johannes L, Wunder C (2011) The SNXy flavours of endosomal sorting. Nat Cell Biol 13:884-886

21. Villar VA, Armando I, Sanada H et al (2013) Novel role of sorting nexin 5 in renal D1 dopamine receptor trafficking and function: implications for hypertension. FASEB J 27:1808-1819

22. Villar VA, Jones JE, Armando I et al (2013) Sorting nexin 1 loss results in D5 dopamine receptor dysfunction in human renal proximal tubule cells and hypertension in mice. J Biol Chem 288:152163

23. Chiu YF, Chuang LM, Hsiao CF et al (2005) An autosomal genome-wide scan for loci linked to pre-diabetic phenotypes in nondiabetic Chinese subjects from the Stanford Asia-Pacific Program of Hypertension and Insulin Resistance Family Study. Diabetes 54:1200-1206

24. Liu H, Liu ZQ, Chen CX, Magill S, Jiang Y, Liu YJ (2006) Inhibitory regulation of EGF receptor degradation by sorting nexin 5. Biochem Biophys Res Commun 342:537-546

25. Otsuki T, Kajigaya S, Ozawa K, Liu JM (1999) SNX5, a new member of the sorting nexin family, binds to the Fanconi anemia complementation group A protein. Biochem Biophys Res Commun 265:630-635

26. Tiwari S, Halagappa VK, Riazi S, Hu X, Ecelbarger CA (2007) Reduced expression of insulin receptors in the kidneys of insulinresistant rats. J Am Soc Nephrol 18:2661-2671

27. Konkalmatt PR, Asico LD, Zhang Y, et al. (2016) Renal rescue of dopamine $\mathrm{D} 2$ receptor function reverses renal injury and high blood pressure. JCI Insight 1:e85888 
28. Ayala JE, Samuel VT, Morton GJ et al (2010) Standard operating procedures for describing and performing metabolic tests of glucose homeostasis in mice. Dis Model Mech 3:525-534

29. Bowe JE, Franklin ZJ, Hauge-Evans AC, King AJ, Persaud SJ, Jones PM (2014) Metabolic phenotyping guidelines: assessing glucose homeostasis in rodent models. J Endocrinol 222:G13-G25

30. Sanada H, Yatabe J, Midorikawa S et al (2006) Amelioration of genetic hypertension by suppression of renal $\mathrm{G}$ protein-coupled receptor kinase type 4 expression. Hypertension 47:1131-1139

31. Li F, Yang J, Jones JE et al (2015) Sorting nexin 5 and dopamine D1 receptor regulate the expression of the insulin receptor in human renal proximal tubule cells. Endocrinology 156:2211-2221

32. Reaven GM, Chang H (1991) Relationship between blood pressure, plasma insulin and triglyceride concentration, and insulin action in spontaneous hypertensive and Wistar-Kyoto rats. Am J Hypertens 4:34-38

33. Potenza MA, Marasciulo FL, Chieppa DM et al (2005) Insulin resistance in spontaneously hypertensive rats is associated with endothelial dysfunction characterized by imbalance between NO and ET-1 production. Am J Physiol Heart Circ Physiol 289: H813-H822

34. Teasdale RD, Loci D, Houghton F, Karlsson L, Gleeson PA (2001) A large family of endosome-localized proteins related to sorting nexin 1. Biochem J 358:7-16

35. Rigalleau V, Blanchetier V, Combe C (1997) A low-protein diet improves insulin sensitivity of endogenous glucose production in predialytic uremic patients. Am J Clin Nutr 65:1512-1516

36. Hulse RE, Ralat LA, Wei-Jen T (2009) Structure, function, and regulation of insulin-degrading enzyme. Vitam Horm 80:635-648
37. Tiganis $\mathrm{T}$ (2011) Reactive oxygen species and insulin resistance: the good, the bad and the ugly. Trends Pharmacol Sci 32:82-89

38. Butlen D, Vadrot S, Roseau S, Morel F (1988) Insulin receptors along the rat nephron: [125I] insulin binding in microdissected glomeruli and tubules. Pflugers Arch 412:604-612

39. Reaven GM, Hoffman BB (1987) A role for insulin in the aetiology and course of hypertension? Lancet 2:435-437

40. Hayashi K, Fujiwara K, Oka K, Nagahama T, Matsuda H, Saruta T (1997) Effects of insulin on rat renal microvessels: studies in the isolated perfused hydronephrotic kidney. Kidney Int 51:1507-1513

41. Hall JE (1993) Hyperinsulinemia: a link between obesity and hypertension? Kidney Int 43:1402-1417

42. Li R, Zhang H, Wang $\mathrm{W}$ et al (2010) Vascular insulin resistance in prehypertensive rats: role of PI3-kinase/Akt/eNOS signaling. Eur J Pharmacol 628:140-147

43. Finch D, Davis G, Bower J, Kirchner K (1990) Effect of insulin on renal sodium handling in hypertensive rats. Hypertension 15:514 518

44. Sechi LA (1999) Mechanisms of insulin resistance in rat models of hypertension and their relationships with salt sensitivity. J Hypertens 17:1229-1237

45. Carey RM, Schoeffel CD, Gildea JJ et al (2012) Salt sensitivity of blood pressure is associated with polymorphisms in the sodiumbicarbonate cotransporter. Hypertension 60:1359-1366

46. Vu PY, Toutain J, Cappellen D et al (2012) A homozygous balanced reciprocal translocation suggests LINC00237 as a candidate gene for MOMO (macrosomia, obesity, macrocephaly, and ocular abnormalities) syndrome. Am J Med Genet A 158A:2849-2856

47. Isomaa B (2003) A major health hazard: the metabolic syndrome. Life Sci 73:2395-2411 